Elkahloun, Abdel

\section{In vivo expression profile analysis of human breast cancer progression using laser microdissection, CDNA array and quantitative real-time PCR}

\author{
Abdel G. Elkahloun ${ }^{1}$, Sarena Teng ${ }^{1}$, Jim Hudson ${ }^{1}$, Greg Robinson ${ }^{2}$, \\ Rebbecca LeVangie ${ }^{3} \&$ Dennis C. Sgroi ${ }^{3}$
}

${ }^{1}$ M icroarray Department, Research Genetics, Inc., Huntsville, Alabama, USA
${ }^{2}$ O pthalmology Research, Children's Hospital, Boston, M assachusetts, USA
${ }^{3}$ M olecular Pathology Unit, M assachusetts General Hospital, Charlestown, and Harvard M edical School, Boston, M assachusetts, USA

The recent advent of high-density cDNA microarray technology, with its capacity for simultaneous monitoring of thousands of genes, provides a unique opportunity for high-throughput genetic analysis of cancer. Although most current microarray studies have been performed with in vitro-derived genetic material, a major leap in functional genomic investigations would be the ability to perform array-based expression analysis with in vivo-derived genetic material originating from morphologically distinct cellular populations, including various stages of cancer progression. Until recently, in vivo analysis of tumour-specific genomic alterations, array-based or otherwise, has been hampered by the inability to accurately obtain specific cell types from cancerous tissue. The recent development of laser capture microdissection (LCM) has allowed for accurate and rapid procurement of specific cell populations in complex tissue and provides the opportunity to perform array-based expression profiling of in vivo-derived genetic material. Here we describe the combined use of LCM, high-throughput cDNA microarrays and Taqman real-time quantitative PCR (RTQ-PCR) to monitor gene expression levels in breast cancer. We demonstrate that expression profiles of greater than 8,000 genes can be successfully generated using non-amplified RNA derived from distinct cell populations in several different morphological stages of human breast cancer.

Erlander, Mark

\section{Using laser capture microdissection (LCM) to build CDNA microarray expres- sion databases with cell-type specificity}

\author{
Mark G. Erlander, Jackson S. W an \& M ichael R. Jackson \\ R.W. Johnson Pharmaceutical Research Institute, \\ San Diego, California 92121, USA
}

Gene expression profiles of thousands of genes can now be examined en masse through cDNA and oligonucleotide microarrays. However, present applications of microarray technology do not include the study of gene expression from individual cell types residing in a given tissue/organ. This kind of resolution is absolutely necessary if one wants to generate hypotheses regarding mechanistic interactions between different cell types. We have previously demonstrated that by integrating laser capture microdissection (LCM) with DNA chips one can obtain cell type gene expression profiles (Luo et al., Nature Medicine, 5, 1999, 177-122). We present details of how we are using LCM to obtain cell-type specificity by giving examples within the brain and peripheral tissues. In addition, we will demonstrate the use of cluster analysis for the identification of genes whose expression is coordinated within specific cell types.
Falciani, Francesco

\section{Monitoring cell response by cluster analysis of a gene expression matrix}

\author{
F. Falciani ${ }^{1}$, J.M. Cox ${ }^{2}$, C.L. Clayton ${ }^{1}$, Phil Robinson ${ }^{2}$, \\ S. Blakemore ${ }^{1}$, D.M. Wallace ${ }^{1}$, J.E. Crabtree ${ }^{2}$, M .K. Trower ${ }^{1}$ \\ \& P. Sanseau ${ }^{1}$ \\ ${ }^{1}$ Genomics Unit, Glaxo Wellcome M edicines Research Centre, \\ Gunnels Wood Rd, Stevenage, Herts., SG1 2NY, UK \\ ${ }^{2}$ M olecular M edicine Unit, St Jame's University Hospital, Leeds, UK
}

We have developed a technology for the identification of genes differentially regulated between biological samples based around the hybridisation of RNAderived probes onto nylon membranes containing immobilised cDNAs gridded at high density. We used this technology to examine changes in gene expression patterns in gastric epithelial cells infected with a virulent $H$. pilory strain at different time points. Radioactively labelled first strand cDNA, synthesised from KATO III gastric cell mRNA, was prepared and hybridised to high density arrays of amplified cDNA derived from a collection of 46,302 non-redundant I.M.A.G.E clones. Hybridisation signals were acquired using the GlaxoWellcome developed DGENT PC-based software package.

The large gene expression matrix generated with this approach has been analysed using cluster analysis to reveal correlated patterns of gene expression. The use of principal component analysis has also been explored. We found that a considerable number of genes map into discrete gene clusters suggesting that they belong to defined pathways of gene expression. We are investigating the possibility of applying this approach to gene expression matrixes based on the frequency of appearance of a gene transcript in representative libraries. Our results reinforce the use of statistical analysis in differential gene expression experiments.

Flores Morales, Amilcar

\section{Differential cloning of growth hormone- regulated hepatic transcripts in the aged rat}

\author{
Petra Tollet-Egnell ${ }^{1}$, Amilcar Flores-M orales ${ }^{1}$, Joakim Lundeberg ${ }^{2}$ \\ \& Gunnar Norstedt ${ }^{1}$
}

It has been suggested that ageing, or at least some of its symptoms, are related to a physiological decline in growth hormone $(\mathrm{GH})$ levels with age. To elucidate the molecular mechanisms behind GH-dependent changes in aged animals, we have analysed GH-mediated effects on gene expression in old male rats through the application of cDNA representational difference analysis in combination with RNase protection assays and DNA macroarrays on nylon filters. In addition to well-characterized GH-regulated genes, we demonstrate the differential expression of at least 11 genes not previously known to be under GH control. Several transcripts encoding enzymes and receptors involved in the metabolism of protein, carbohydrates and lipids were identified. Another group of transcripts consisted of gene products encoding proteins involved in the immune system. This list of expressed genes in GH-treated old rats may shed further insight on the action and mechanism behind the positive effects of $\mathrm{GH}$ on, for example, body composition and immune responses observed in different animal and human studies. 